\title{
TORSION IN THE BORDISM OF ORIENTED INVOLUTIONS
}

\author{
BY
}

RUSSELL J. ROWLET'

\begin{abstract}
In the bordism theory $\Omega_{*}\left(Z_{2}^{k}\right)$ of smooth, orientation-preserving $Z_{2}^{k}$-actions all torsion has order two. Furthermore, the torsion classes inject in the unoriented theory $N_{*}\left(Z_{2}^{k}\right)$, and any class represented by a stationarypoint free action has infinite order. In addition, a procedure is given for producing Smith constructions in some generality.
\end{abstract}

1. Introduction. It is well known that the only torsion in the oriented bordism ring $\Omega_{*}$ is of order two [13]. The same is true of the bordism of smooth, orientation-preserving involutions [5]. We shall show that for every $k \geqslant 0$ the torsion in the bordism of smooth, orientation-preserving $Z_{2}^{k}$-actions is all of order two.

We adopt the notation of R. E. Stong [10], [11] for equivariant bordism theories. In particular, $\Omega_{*}\left(Z_{2}^{k}\right)$ is the bordism of oriented $Z_{2}^{k}$-actions. Recall that a collection $\mathscr{F}$ of subgroups of $Z_{2}^{k}$ is a family if $K \in \mathscr{F}$ whenever $H \in \mathscr{F}$ and $K$ is a subgroup of $H$, written $K \leqslant H$. In particular, there is the family $\mathscr{P}$ of proper subgroups of $Z_{2}^{k}$. Then $\Omega_{*}\left(Z_{2}^{k}, \mathcal{P}\right)$ denotes the bordism of oriented, stationary-point free $Z_{2}^{k}$-actions.

THEOREM. All torsion of $\Omega_{*}\left(Z_{2}^{k}\right)$ is of order two. Furthermore, the forgetful homomorphism $\Omega_{*}\left(Z_{2}^{k}, \mathcal{P}\right) \rightarrow \Omega_{*}\left(Z_{2}^{k}\right)$ has torsionfree image.

The second statement is the oriented analogue of Stong's theorem [12, Proposition 2] that in unoriented bordism a stationary-point free $Z_{2}^{k}$-action always bounds.

The rest of this note is devoted to the proof of the theorem. One or two of its features may be of independent interest. We need an equivariant version of Burdick's theorem (which is the isomorphism $\left.\tilde{\Omega}_{*}\left(B Z_{2}\right)=N_{*-1}\right)$. This requires that we be able to construct invariant submanifolds dual, in an appropriate sense, to a representation of $Z_{2}^{k}$. Similar constructions have proliferated in equivariant differential topology since Conner and Floyd first defined the

Received by the editors December 13, 1974 and, in revised form, February 10, 1976.

AMS (MOS) subject classifications (1970). Primary 57D85, Secondary 57D15.

Key words and phrases. Orientation-preserving involution, Wall manifold, submanifold dual to a representation. 
Smith construction $[2, \S 26]$. We shall prove a lemma that provides such constructions in general, in the hope that it will cover future needs as well as past examples.

The theorem was announced in [8], along with some further claims, which are not true. I am much indebted to Gary Hamrick for discovering the error in time to prevent publication of the erroneous proof.

2. Outline of the argument. If $H \leqslant Z_{2}^{k}$, let us write $\mathbb{Q}(H)$ for the family of all subgroups of $H$. We make the abbreviation $\mathscr{Q}=\mathscr{Q}\left(Z_{2}^{k}\right)$, so that $\Omega_{*}\left(Z_{2}^{k}\right)$ $=\Omega_{*}\left(Z_{2}^{k}, \mathbb{Q}\right)$.

PROPOSITION 1. All torsion of the relative bordism group $\Omega_{*}\left(Z_{2}^{k}, \mathfrak{Q}, \mathcal{P}\right)$ is of order two.

Proof. If $\phi: Z_{2}^{k} \times M \rightarrow M$ represents an element of $\Omega_{*}\left(Z_{2}^{k}, \mathcal{Q}, \mathcal{P}\right)$, then a tubular neighborhood of the fixed set $X$ of $\phi$ will represent the same element $[11, \S 5]$. Suppose $X_{0}$ is a component of $X$, and let $\left\{R_{i}\right\}$ be the set of nontrivial irreducible real representations of $Z_{2}^{k}$. Then the normal bundle $\nu$ of $X_{0}$ in $M$ decomposes:

$$
\nu=\sum_{i} \xi_{i} \otimes R_{i}
$$

according to $[2, \S 38]$.

Let $\gamma(m) \rightarrow B O(m)$ be the universal $m$-plane bundle. For a suitable sequence $I=\left(i_{1}, \ldots, i_{q}\right)$ of nonnegative even integers $\left(q=2^{k}-1\right.$ and $\sum_{j} i_{j}$ $=n$ ), the bundle $\nu$ will be classified by a bundle mapping into

$$
\gamma^{I}=\gamma\left(i_{1}\right) \times \cdots \times \gamma\left(i_{q}\right) \rightarrow B O\left(i_{1}\right) \times \cdots \times B O\left(i_{q}\right) .
$$

Therefore $\Omega_{n}\left(Z_{2}^{k}, \mathcal{Q}, \mathcal{P}\right)$ is isomorphic to the direct sum (over all such sequences $I)$ of the bordism groups $\Omega_{n}\left(D \gamma^{I}, S \gamma^{I}\right)$. To finish the proof, apply $[2,(15.2)]$ and the Künneth theorem, as in the proof of [5, (3.4)].

To prove the theorem, it suffices to show that the forgetful homomorphism $\Omega_{*}\left(Z_{2}^{k}, \mathbb{Q}\right) \rightarrow \Omega_{*}\left(Z_{2}^{k}, \mathbb{Q}, \mathscr{P}\right)$ is injective on the torsion of $\Omega_{*}\left(Z_{2}^{k}, \mathbb{Q}\right)$, and then apply the exactness theorem [10, Proposition 2.2] for the pair $(\mathbb{Q}, \mathcal{P})$ of families. To do this, we shall need to assume that the theorem is true for actions of $Z_{2}^{k-1}$. We shall define a sequence of families

$$
\varnothing=\mathscr{F}_{0} \subset \mathscr{F}_{1} \subset \mathscr{F}_{2} \subset \cdots \subset \mathscr{F}_{m}=\mathscr{P} \subset \mathbb{Q}
$$

of subgroups of $Z_{2}^{k}$. We shall show that the homomorphisms

$$
\Omega_{*}\left(Z_{2}^{k}, \mathscr{F}_{i}, \mathscr{F}_{i-1}\right) \rightarrow \Omega_{*}\left(Z_{2}^{k}, \mathcal{Q}, \mathscr{F}_{i-1}\right) \quad(i=1,2, \ldots, m)
$$

have torsionfree image. It follows that the homomorphisms 


$$
\Omega_{*}\left(Z_{2}^{k}, \mathcal{Q}, \mathscr{F}_{i-1}\right) \rightarrow \Omega_{*}\left(Z_{2}^{k}, \mathcal{Q}, \mathscr{F}_{i}\right) \quad(i=1,2, \ldots, m)
$$

are injective on torsion, and thus that

$$
\Omega_{*}\left(Z_{2}^{k}\right)=\Omega_{*}\left(Z_{2}^{k}, \mathcal{Q}, \mathscr{F}_{0}\right) \rightarrow \Omega_{*}\left(Z_{2}^{k}, \mathcal{Q}, \mathscr{F}_{m}\right)=\Omega_{*}\left(Z_{2}^{k}, \mathcal{Q}, \mathcal{P}\right)
$$

is injective on torsion.

3. A suitable sequence of families. We now choose a sequence (1) of families of $Z_{2}^{k}$. The first step is to list the subgroups $H_{1}, H_{2}, \ldots, H_{m}$ of $Z_{2}^{k}$ of order $2^{k-1}$. Unfortunately, this cannot be done at random.

Choose, once and for all, a basis $\left\{t_{1}, \ldots, t_{k}\right\}$ of $Z_{2}^{k}$. If $H<Z_{2}^{k}$ has order $2^{k-1}$, define an integer $a(H)$ as follows: $a(H)=\sup \left\{j: t_{1}, \ldots, t_{j} \in H\right\}$. The subgroups $H_{1}, \ldots, H_{m}$ are to be listed so that $a\left(H_{i+1}\right) \leqslant a\left(H_{i}\right)$ for each $i=1, \ldots, m-1$. Thus $H_{1}$ is generated by $t_{1}, \ldots, t_{k-1}$, while $H_{2}$ can be any other subgroup of order $2^{k-1}$ which contains $t_{1}, \ldots, t_{k-2}$.

The sequence (1) is defined by the rule $\mathscr{F}_{i}=\mathscr{F}_{i-1} \cup \mathbb{Q}\left(H_{i}\right)$, for $i=1,2, \ldots$, $m$. Clearly, $\mathscr{F}_{m}=\mathscr{P}$. Our care in constructing (1) produces the following:

Proposition 2. Suppose $1<i \leqslant m$. If $K \in \mathscr{F}_{i-1} \cap \mathbb{Q}\left(H_{i}\right)$ and $a\left(H_{i}\right)=j$, then the subgroup $\bar{K}$, generated by $K$ and $t_{j+1}$, belongs to $\mathscr{F}_{i-1}$.

Proof. By hypothesis, $K \leqslant H \cap H_{i}$ for some subgroup $H$ having $a(H)$ $\geqslant j$. Since $H \cap H_{i}$ is a subgroup of order $2^{k-2}$, containing $t_{1}, \ldots, t_{j}$ but not $t_{j+1}$, we may as well assume that $t_{j+1} \in H$. Then $a(H)>j$ and $\bar{K} \leqslant H$, so $\bar{K} \in \mathscr{F}_{i-1}$.

Proposition 3. If $G$ is an abelian group, $K \leqslant G$ is a subgroup, $\mathscr{F}^{\prime} \subset \mathcal{F}$ are families of subgroups, and every member of $\mathscr{F}-\mathscr{F}^{\prime}$ is contained in $K$, then the inclusion $\left(\mathscr{F} \cap \mathbb{Q}(K), \mathscr{F}^{\prime} \cap \mathbb{Q}(K)\right) \subset\left(\mathscr{F}, \mathscr{F}^{\prime}\right)$ induces an isomorphism

$$
\Omega_{*}\left(G, \mathscr{F}, \mathscr{F}^{\prime}\right) \cong \Omega_{*}\left(G, \mathscr{F} \cap \mathbb{Q}(K), \mathscr{F}^{\prime} \cap \mathbb{Q}(K)\right) .
$$

In particular, we have the isomorphism

$$
\Omega_{*}\left(Z_{2}^{k}, F_{i}, F_{i-1}\right) \cong \Omega_{*}\left(Z_{2}^{k}, A\left(H_{i}\right), F_{i-1} \cap A\left(H_{i}\right)\right) .
$$

Proof. This seems clear enough, but I cannot find a suitable lemma in the literature. If $\mathscr{F}-\mathscr{F}^{\prime}$ consists of a single subgroup $K^{\prime}$, then the proposition follows from [11, Lemma 5.1, p. 14] by discarding all but a tubular neighborhood of the fixed set of $K^{\prime}$. Now we can easily define families $\mathscr{F}^{\prime}=\mathfrak{L}_{0} \subset \mathfrak{L}_{1}$ $\subset \cdots \subset \mathfrak{L}_{h}=\mathscr{F}$ such that each $\mathfrak{L}_{j}-\mathfrak{L}_{j-1}$ is a singleton. The reader can then prove by induction on $j$ that

$$
\Omega_{*}\left(G, \mathscr{L}_{j}, \mathscr{F}^{\prime}\right) \cong \Omega_{*}\left(G, \mathfrak{L}_{j} \cap \mathbb{Q}(K), \mathscr{F}^{\prime} \cap \mathbb{Q}(K)\right),
$$

using exactness [10, Proposition 2.2] and the 5-lemma. 
4. Smith constructions and Burdick's theorem. The next task is to consider the right side of (2). We recall Burdick's theorem [3, p. 155], which gives an isomorphism

$$
\Omega_{m}\left(Z_{2},\{\{1\}\}\right) \cong \Omega_{m} \oplus N_{m-1} .
$$

We shall generalize this to $Z_{2}^{k}$-actions (Proposition 5). First it is necessary to look at a question of rather general interest.

Let the finite group $G$ act smoothly on the compact, smooth manifold $M$. Suppose $\theta: G \times R^{n} \rightarrow R^{n}$ is an orthogonal representation. An invariant submanifold $N \subset M$, of codimension $n$, is dual to $\theta$ if its normal bundle in $M$ has the form $R^{n} \times N \rightarrow N$, where $G$ acts on $R^{n}$ via $\theta$ and on $N$ by the restriction of its action on $M$. For arbitrary $M$ and $\theta$, we have no reason to think that such a submanifold exists.

Proposition 4. Suppose $G$ is nilpotent, $K$ is a normal subgroup of $G$, every isotropy group of the $G$-manifold $M$ is a subgroup of $K$, and $K \leqslant \operatorname{Ker} \theta$. Then there is an invariant submanifold $N \subset M$ dual to $\theta$. Furthermore, for families $\mathscr{F}^{\prime} \subset \mathscr{F} \subseteq \mathbb{Q}(K)$ there is a homomorphism

$$
\Delta(\theta): N_{*}\left(G, \mathscr{F}, \mathscr{F}^{\prime}\right) \rightarrow N_{*}\left(G, \mathscr{F}, \mathscr{F}^{\prime}\right)
$$

of degree $-n$, such that $\Delta(\theta)[M]$ is represented by a submanifold dual to $\theta$.

REMARK. If $G=Z_{2}$ and $\theta$ is the nontrivial representation of $Z_{2}$ on $R$, then $\Delta(\theta)$ is the Smith homomorphism of Conner and Floyd $[2, \S 26]$. All the other "Smith constructions" found in the literature, so far as the author knows, are covered by the proposition, or its obvious analogues in other bordism theories.

Proof. We use the terminology of G. Bredon [1]. Let $\Sigma$ be the collection of all orbit types $G / H \leqslant G / K$. By a famous theorem of R. S. Palais [4], there is a $G$-space $E=E(\operatorname{dim} M, \Sigma)$ such that $M$ admits an isovariant map $f_{M}: M$ $\rightarrow E$ which is unique up to $G$-isovariant homotopy. Bredon shows $[1$, p. 111] that we can equip $E$ with a standard equivariant embedding $u: E \rightarrow R^{q}-\{0\}$ where the action of $G$ on $R^{q}-\{0\}$ is orthogonal.

Thus we can assume that in some neighborhood $U$ of $\operatorname{Im} u$ all orbit types are $\leqslant G / K$. Give $R^{n} \times U$ the product $G$-action and consider the $G$-equivariant map

$$
g_{M}: M \stackrel{f_{M}}{\longrightarrow} E \stackrel{u}{\longrightarrow} U \rightarrow\{0\} \times U \rightarrow R^{n} \times U
$$

By [7, Lemma 1(b)] we can deform $g_{M}$ by a $G$-homotopy to a map $g_{M}^{\prime}$ transverse to $\{0\} \times U \subset R^{n} \times U$. Then $g_{M}^{\prime-1}(\{0\} \times U)=N$ is dual to $\theta$ in $M$.

If $M$ has nonempty boundary, we must first deform $g_{M}$ so that on a collar neighborhood $\partial M \times[0,1)$ it factors through the projection on $\partial M$; then we 
make $g_{\partial M}$ transverse to $\{0\} \times U$ and extend in the obvious way so that $g_{M}$ is transverse for points in a collar neighborhood. Finally we deform $g_{M}$ to be transverse for all points of $M$, leaving it fixed on $\partial M$. If $M$ is an $\left(\mathscr{F}, \mathscr{F}^{\prime}\right)-$ bordism between $V_{1}$ and $V_{2}$, say, then the restriction $g_{M} \mid V_{i}$ is isovariantly homotopic to $g_{V_{i}}$. Thus the resulting $g_{M}^{\prime}$ will give a bordism of $\Delta(\theta)\left(V_{1}\right)$ to $\Delta(\theta)\left(V_{2}\right)$. This completes the proof.

Next, let $Z_{2}^{k}=H \times Z_{2}$ for some subgroup $H$ of order $2^{k-1}$. Let $\left(\mathscr{F}, \mathscr{F}^{\prime}\right)$ be families, $\mathscr{F}^{\prime} \subset \mathscr{F} \subseteq \mathbb{Q}(H)$. Let $\theta: Z_{2}^{k} \times R \rightarrow R$ be the nontrivial representation with kernel $H$. Suppose $[M, \phi] \in \Omega_{m}\left(Z_{2}^{k}, \mathscr{F}, \mathscr{F}^{\prime}\right)$. Let $N \subset M$ be dual to $\theta$, and let $N_{1}=N / Z_{2}$. Since $Z_{2}$ will reverse the orientation of $N$, the projection $p: N \rightarrow N_{1}$ is the oriented double cover of $N_{1}$.

Let $\lambda \rightarrow N_{1}$ be the real line bundle associated to $N \rightarrow N_{1}$. Then $\lambda$ is a $Z_{2}^{k}$ equivariant bundle, and the sphere bundle $\pi: S(\lambda \oplus R) \rightarrow N_{1}$ admits an orientation-preserving $Z_{2}^{k}$-action, given by the product of the action on $\lambda$ and the action $\theta$ on $R$.

Suppose $t$ is a generator of $Z_{2}$. If $g x=x$ for $g \in Z_{2}^{k}, x \in S(\lambda \oplus R)$, then we may write $g=h t^{e}$ for some $h \in H$. Clearly $h \pi(x)=\pi(x) \in N_{1}$. Because $\mathscr{F} \subseteq \mathbb{Q}(H)$ it is easy to verify that $h\left(x^{\prime}\right)=x^{\prime} \in N$ for $p\left(x^{\prime}\right)=\pi(x)$; thus $G_{x} \leqslant G_{x^{\prime}}$ and $G_{x} \in \mathscr{F}$. This shows that the action on $S(\lambda \oplus R)$ is $\left(\mathscr{F}, \mathscr{F}^{\prime}\right)$-free. By Proposition 4, the assignment of $S(\lambda \oplus R)$ to $M$ gives a homomorphism

$$
\sigma: \Omega_{m}\left(G, \mathscr{F}, \mathscr{F}^{\prime}\right) \rightarrow \Omega_{m}\left(G, \mathscr{F}, \mathscr{F}^{\prime}\right)
$$

Since $S \lambda=\Delta(\theta)(S(\lambda \oplus R))$, we have $\sigma^{2}=\sigma$.

In the original version of Burdick's theorem, with $H=1$ and all $Z_{2}$-actions free, assigning $N_{1}$ to $M$ gives the epimorphism $\Omega_{m}\left(Z_{2},\{\{1\}\}\right) \rightarrow N_{m-1}$. At our level of generality, $\Omega_{m}\left(Z_{2}^{k}, \mathscr{F}, \mathscr{F}^{\prime}\right) \rightarrow N_{m-1}\left(Z_{2}^{k-1}, \mathscr{F}, \mathscr{F}^{\prime}\right)$ is not known to be a surjective. We can, however, prove the following.

Proposition 5 (BURdick's THEOREM). As before, let $Z_{2}^{k}=H \times Z_{2}$ and suppose $\mathscr{F}^{\prime} \subset \mathscr{F} \subseteq \mathbb{Q}(H)$. Let $e: \Omega_{m}\left(H, \mathscr{F}, \mathscr{F}^{\prime}\right) \rightarrow \Omega_{m}\left(Z_{2}^{k}, \mathscr{F}, \mathscr{F}^{\prime}\right)$ be the extension homomorphism. Then $e$ is a monomorphism, and $\Omega_{m}\left(Z_{2}^{k}, \mathscr{F}, \mathscr{F}^{\prime}\right) \cong \operatorname{Im} e \oplus \operatorname{Im} \sigma$.

Proof. If $[P, \psi] \in \Omega_{m}\left(Z_{2}^{k-1}, \mathscr{F}, \mathscr{F}^{\prime}\right)$, then $e[P, \psi]$ is represented by $P \times S^{0}$ with the obvious action: $Z_{2}$ acts trivially on $P$ and reverses the two points of $S^{0}$, while $H$ acts by $\psi \times 1$. This is clearly split by assigning $M / Z_{2}$ to each $Z_{2}^{k}$ action on $M$. Since $\sigma^{2}=\sigma$, the inclusion $\operatorname{Im} \sigma \subset \Omega_{m}\left(Z_{2}^{k}, \mathscr{F}, \mathscr{F}^{\prime}\right)$ is also split.

Now suppose $[M, \psi] \in \Omega_{m}\left(Z_{2}^{k}, \mathscr{F}, \mathscr{F}^{\prime}\right)$, and $\sigma[M, \psi]$ is the class of $S(\lambda \oplus R)$, as above. Form $M \times I$ and $S(\lambda \oplus R) \times I$; sew a tubular neighborhood of $N \subset M \times\{1\}$ to a tubular neighborhood of $N=S \lambda \subset S(\lambda \oplus R) \times\{0\}$. The resulting manifold is a cobordism of $M$ to $e\left(M / Z_{2}\right)+S(\lambda \oplus R)$. This completes the proof. 
5. The proof of the theorem. Recall that we need only show that the homomorphism

$$
q: \Omega_{*}\left(Z_{2}^{k}, \mathbb{Q}\left(H_{i}\right), \mathscr{F}_{i-1} \cap \mathbb{Q}\left(H_{i}\right)\right) \cong \Omega_{*}\left(Z_{2}^{k}, \mathscr{F}_{i}, \mathscr{F}_{i-1}\right) \rightarrow \Omega_{*}\left(Z_{2}^{k}, \mathbb{Q}, \mathscr{F}_{i-1}\right)
$$

has torsionfree image. By Proposition 5, we must show that

$$
q e: \Omega_{*}\left(H_{i}, \mathbb{Q}\left(H_{i}\right), \mathscr{F}_{i-1} \cap \mathbb{Q}\left(H_{i}\right)\right) \rightarrow \Omega_{*}\left(Z_{2}^{k}, \mathbb{Q}, \mathscr{F}_{i-1}\right)
$$

and

$$
q \sigma: \Omega_{*}\left(Z_{2}^{k}, \mathscr{Q}\left(H_{i}\right), \mathscr{F}_{i-1} \cap \mathbb{Q}\left(H_{i}\right)\right) \rightarrow \Omega_{*}\left(Z_{2}^{k}, \mathfrak{Q}, \mathscr{F}_{i-1}\right)
$$

have torsionfree image.

We first observe that $q \sigma=0$. Certainly $S(\lambda \oplus R)$ bounds the disk bundle $D(\lambda \oplus R)$; it remains to verify that $D(\lambda \oplus R)$ is $\left(Q, \mathscr{F}_{i-1}\right)$-free. Put $a\left(H_{i}\right)=j$, and $Z_{2}^{k}=H \times Z_{2}$ with $Z_{2}$ generated by $t_{j+1}$. Let $\lambda^{\prime}=\lambda \mid \partial N_{1}$. Each isotropy group $K$ of $S\left(\lambda^{\prime} \oplus R\right)$ lies in $\mathscr{F}_{i-1} \cap \mathbb{Q}\left(H_{i}\right)$; the only new isotropy groups in $D\left(\lambda^{\prime} \oplus R\right)$ will be subgroups of $\bar{K}$, the subgroup generated by $K$ and $t_{j+1}$. By Proposition 2, all such $\bar{K}$ lie in $\mathscr{F}_{i-1}$, which implies $q \sigma=0$.

By induction on $k$, the torsion classes of $\Omega_{m}\left(H_{i}, \mathcal{Q}\left(H_{i}\right), \mathscr{F}_{i-1} \cap \mathbb{Q}\left(H_{i}\right)\right)$ all have order two; one should note here that the sequence

$$
\varnothing \subset \mathscr{F}_{1} \cap \mathbb{Q}\left(H_{i}\right) \subseteq \mathscr{F}_{2} \cap \mathbb{Q}\left(H_{i}\right) \subseteq \cdots \subseteq \mathscr{F}_{m} \cap \mathbb{Q}\left(H_{i}\right)
$$

is the analogue of (1) for $Z_{2}^{k-1}$, except that it has repetitions. Thus it will suffice to show that

$$
q e: \Omega_{m}\left(H_{i}, \mathscr{Q}\left(H_{i}\right), \mathscr{F}_{i-1} \cap \mathbb{Q}\left(H_{i}\right)\right) \rightarrow \Omega_{m}\left(Z_{2}^{k}, \mathcal{Q}, \mathscr{F}_{i-1}\right)
$$

has its kernel equal to the 2-torsion.

Let $r: \Omega_{m}\left(Z_{2}^{k}, \mathbb{Q}, \mathscr{F}_{i-1}\right) \rightarrow \Omega_{m}\left(H_{i}, \mathbb{Q}\left(H_{i}\right), \mathscr{F}_{i-1} \cap \mathbb{Q}\left(H_{i}\right)\right)$ be the restriction of a $Z_{2}^{k}$-action to an $H_{i}$-action. Now rqe is multiplication by 2 , so $\mathrm{Ker} q e$ contains only classes of order two.

We will show that $q e(x)=0$ for every class $x$ of order two. The equivariant Wall bordism theory of [6] is useful for investigating such questions.

If $G$ is a finite group, a smooth $G$-action on $M$ is said to preserve Wall structure if there is a map $f: M \rightarrow S^{1}$ satisfying (i) $f(g m)=f(m)$ for all $g \in G$ and $m \in M$, and (ii) if $\alpha \in H\left(S^{1} ; Z_{2}\right)$ is the nonzero class, then $f^{*} \alpha$ is the Stiefel-Whitney class $w_{1} M$. For each pair of families $\left(\mathscr{F}, \mathscr{F}^{\prime}\right)$ there is a bordism theory $W_{*}\left(G, \mathscr{F}, \mathscr{F}^{\prime}\right)$ of such $G$-actions.

Assume $G$ is nilpotent. By [7, Lemma 1(b)] we can assume $f$ is transverse to some point $* \in S^{1}$. Assigning $f^{-1}(*)$ to $M$ gives a homomorphism $d$ : $W_{m}\left(G, \mathscr{F}, \mathscr{F}^{\prime}\right) \rightarrow \Omega_{m-1}\left(G, \mathscr{F}, \mathscr{F}^{\prime}\right)$. The image of $d$ is precisely the torsion of order 
two in $\Omega_{m-1}\left(G, \mathscr{F}, \mathscr{F}^{\prime}\right)$; the proof is exactly like that of [6, Theorem 4.2].

Consider the extension

$$
\text { qe: } W_{m}\left(H_{i}, \mathcal{Q}\left(H_{i}\right), \mathscr{F}_{i-1} \cap \mathfrak{Q}\left(H_{i}\right)\right) \rightarrow W_{m}\left(Z_{2}^{k}, \mathfrak{Q}, \mathscr{F}_{i-1}\right) .
$$

If $[P, \psi] \in W_{m}\left(H_{i}, \mathbb{Q}\left(H_{i}\right), \mathscr{F}_{i-1} \cap \mathbb{Q}\left(H_{i}\right)\right)$, with $f: P \rightarrow S^{1}$ describing the Wall structure, then $q e[P, \psi]$ is represented by $P \times S^{0}=\partial(P \times[-1,1])$. Clearly $Z_{2}^{k}$ preserves the Wall structure $P \times[-1,1] \rightarrow P \stackrel{f}{\rightarrow} S^{1}$. On $\partial P \times[-1,1]$ the isotropy groups are subgroups of $\bar{K}$, where $K \in \mathscr{F}_{i-1} \cap \mathbb{Q}\left(H_{i}\right)$. Thus $q e=0$, on the Wall bordism. But since $d$ commutes with $e$, this implies that $q e=0$ on the oriented bordism as well. (The argument does not apply directly in the oriented case, because $t_{j+1}$ would reverse the orientation of $P \times[-1,1]$.)

By Proposition 5, we have now shown that the kernel of $q$ is exactly the elements of order two. As outlined in $\$ 2$, this completes the proof of the theorem.

COROLLARY. The homomorphism $\Omega_{*}\left(Z_{2}^{k}\right) \rightarrow N_{*}\left(Z_{2}^{k}\right)$, which forgets orientation, is injective on torsion.

Proof. Consider the diagram

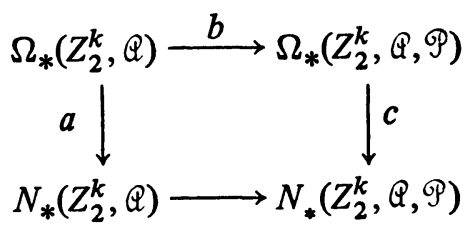

We have shown that $b$ is injective on torsion. Since $c$ is a direct sum of homomorphisms of the form $\Omega_{*}\left(D \gamma^{I}, S \gamma^{I}\right) \rightarrow N_{*}\left(D \gamma^{I}, S \gamma^{I}\right)$, and all torsion in $\Omega_{*}\left(D \gamma^{I}, S \gamma^{I}\right)$ has order 2 , it follows from the Wall sequence [9, p. 169] of $\left(D \gamma^{I}, S \gamma^{I}\right)$ that $c$ is injective on torsion. Thus $a$ is injective on torsion, which was to be proved.

\section{REFERENCES}

1. G. E. Bredon, Introduction to compact transformation groups, Pure and Appl. Math., vol. 46, Academic Press, New York, 1972.

2. P. E. Conner and E. E. Floyd, Differentiable periodic maps, Ergebnisse Math. Grenzgebiete,

Bd. 33; Springer-Verlag, Berlin; Academic Press, New York, 1964. MR 31 \#750.

3. F. Hirzebruch, Involutionen auf Mannigfaltigkeiten, Proc. Conf. Transformation Groups (New Orleans, 1967), Springer, New York, 1968, pp. 148-166. MR 40 \#3578.

4. R. S. Palais, The classification of G-spaces, Mem. Amer. Math. Soc. No. 36 (1960).

5. H. L. Rosenzweig, Bordism of involutions on manifolds, Illinois J. Math. 16 (1972), 1-10.

6. R. J. Rowlett, Wall manifolds with involution, Trans. Amer. Math. Soc. 169 (1972), 153-162. MR 47 \#2628.

7. _ Bounding a free action of a dihedral group, Michigan Math. J. 21 (1974), 363-370. 
8. - Torsion in the bordism of oriented involutions, Notices Amer. Math. Soc. 22 (1975), A227. Abstract \#720-57-1.

9. R. E. Stong, Notes on cobordism theory, Princeton Univ. Press, Princeton, N.J.; Univ. of Tokyo Press, Tokyo, 1968. MR 40 \#2108.

10. - Complex and oriented equivariant bordism, Topology of Manifolds (Proc. Inst., Univ. of Georgia, Athens, 1969), Markham, Chicago, 1970, pp. 291-316. MR 42 \#8521.

11. Unoriented bordism and actions of finite groups, Mem. Amer. Math. Soc. No. 103 (1970). MR 42 \#8522.

12. - Equivariant bordism and $\left(Z_{2}\right)^{k}$ actions, Duke Math. J. 37 (1970), 779-785. MR 42 \#6847.

13. C. T. C. Wall, Determination of the cobordism ring, Ann. of Math. (2) 72 (1960), 292-311. MR 22 \#11403.

Department of Mathematics, University of Tennessee, Knoxville, Tennessee 37916 